International Journal of Applied Mathematical Research, $8(1)(2019) 20-26$
International Journal of Applied Mathematical Research
SPC
Website $:$ www.sciencepubco.com/index.php/IJAMR
Research paper

\title{
Comparison of query optimization techniques in content based image retrieval (CBIR)
}

\author{
Mariam Alharthi ${ }^{1}$, Fahad Alqurashi ${ }^{2} *$ \\ ${ }^{1}$ Department of Computer Science, FCIT, King Abdulaziz University, Saudi Arabia \\ ${ }^{2}$ Information Technology for Infrastructure, King Abdulaziz University, Saudi Arabia \\ *Corresponding authorE-mail: fahad@kau.edu.sa
}

\begin{abstract}
With increasing the popularity of World Wide Web, storing digital contents increases enormously, in that case, it is important to implement convenient information systems which manage the collections of these digital contents efficiently. This paper concentrates on hastening techniques for efficient retrieval of images. Content-Based Image Retrieval (CBIR) systems are used by common approaches. These systems support retrieving similar images depend on content properties (e.g., color, shape, and texture) by retrieving automatically similar images to a pattern or user-defined specification. The CBIR generally used in several applications by applying different techniques in each application which in turns enhance the retrieval process. The paper aims to evaluate some of these applications and compare them to find out the proper methods that return the best results in these CBIR systems.
\end{abstract}

Keywords: Content-Based Image Retrieval; CBIR; Query Optimization; A Multimedia Database; Image Retrieval.

\section{Introduction}

The development in data storage and computing technologies made the demand for multimedia databases. In order to handle these data, it is necessary to use a query optimizer that helps speeding up the retrieval process. One of the important services needs a support from these systems is an image searching. There are two approaches used in image searching, which are: search based on textual metadata and search based on content information.

In first approach, textual metadata is attached to each image in the database in order to use the same query retrieval techniques that are used in traditional databases which simply based on keywords. But this annotation process is a time-consuming task. Also, the annotation taken from users may be inefficient because different users have different viewpoints to describe the same image. All these factors decrease the performance of such systems that used this approach.

To overcome these shortcomings, the second approach which is Content-Based Image Retrieval (CBIR) has emerged. In these CBIR systems, there are many image processing algorithms which are used in order to extract the image properties that represented by feature vectors (e.g. shape, color, and texture). Therefore, the retrieval process in these systems can be done automatically unlike the first approach. This paper aims to analyze the performance of some CBIR systems that uses different models and compare them to describe the effectiveness of certain factors in these systems and presenting the state of art for the existing query optimization techniques in multimedia databases.

The paper explains some basic concepts related to multimedia databases, query processing and query optimization techniques in Section II. In Section III, an overview of CBIR architecture and existing applications are presented. Section IV gives a short review of existing approaches in a CBIR field. Whereas sections V and VI present the current problem and analyzing some of the existing methods used in CBIR systems. In section VII, the experiments were conducted to compare between some CBIR software. Finally, the last two sections VIII and IX conclude the overall work and produce the future work respectively.

\section{Basic concepts}

In this section, several notions related to querying on multimedia databases are explained. Firstly, section A describes the multimedia databases. In the following, the query processing phases in such databases and the optimization techniques that can enhance and speed up the query retrieval process are presented in sections B and C respectively.

\subsection{Multimedia databases}

The Multimedia database is a combination of related multimedia data that contains one or more basic media data types such as images, text, audio, video, graphic objects and animation. The graphic objects involve sketches, drawings, and illustrations [1]. 
A Multimedia Database Management System (MMDBMS) manages multiple data types that represented in different formats. It facilitates the creation, storing, accessing, query and controlling of a multimedia database [1]. Simultaneously, adhere to numerical constraints that are not found in traditional databases. The differences from traditional databases lie in data types, manipulation, storage, and delivery. MMDBs are providing all the functionalities that a traditional database provides, whereas MMDB provides some new and improved functionalities and features [2].

Retrieval in multimedia databases can be categorized into two types: attribute-based and content-based. The queries in these databases are processed in several stages that shown in Fig. 1 [3].

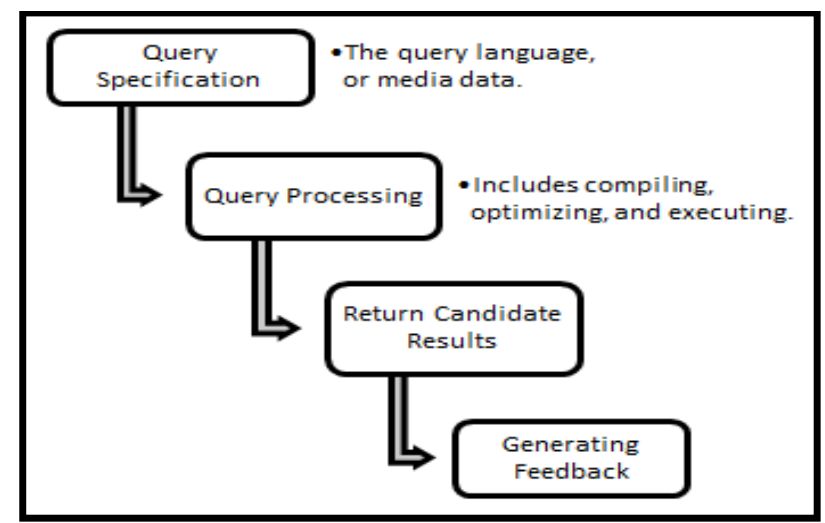

Fig. 1: Query Processing Stages in Multimedia Databases.

\subsection{Query processing}

In traditional database management systems, the users request information in a direct way, then, the results from tables are returned. This shows that the method used to retrieve the queries is attribute-based. On the other hand, in multimedia databases, there is another method that is used to retrieve a query, which is the content-based method. As shown in Fig. 1, the query processing passes through several stages. A description of these stages is as following:

Query specification: the media data or query language, which includes fuzzy manipulation to proceed nearest neighbor searches, proximity searches, to carry out content-based searches. There are four aspects in query specification which are: visual queries by example, incertitude (using "similar with" attribute, the argument may be a complex image), choice ranges (e.g. color, texture etc.) and weights [3].

Query processing: a query is compiled, optimized and executed to obtain the results. The complicated part is the optimization with respect to weights and fuzzy terms [3].

Return candidate results: weights and object attribute values of a query used to calculate the candidate results that are submitted later to the users. Where these results must listed quickly with a good quality also [3].

Generating feedback: can be found in advanced retrieval systems after present the results, which is the user modification of the query. As well as, the actions taken by a system like adding or deleting terms to predicates and re-weighting the terms of initial predicate too. These refinements are vital due to an ambiguity of objects' representation and to a complexity of the object space [3].

\subsubsection{Query optimization techniques}

The Query optimization in multimedia databases becomes the most important issue in academic and business fields [4]. There are different types of techniques depend on multimedia object type [5]. The most common optimization techniques are as follows:

Content-Based Retrieval (CBR)

CBR technique is the communistic one. The queries are used to access semantic information included in multimedia data like video data and motion information [6]. A retrieval process takes advantages of texture, shape, color, and other properties from raw data to analyze this data in respect meaningful entities and geometric patterns [7]. Using CBR in image retrieval requires high level semantics from human to evaluate the retrieval results, hence, overcome the semantic gap in image and video objects [8]. "Similarity search" is one of the essential implementation in a classification of CBR. There are two types of similarity queries: the first is whole matching, which means the compared sequences have the same length [9]. The second is sequence matching, where the sequence of a query is smaller and the comparison done in large sequence to obtain the best match [9]. This similarity search method is appropriate to be used.

\subsubsection{Semantic based retrieval}

The semantic query according to [10] uses knowledge about nature of data, the domain of relations and constraints related to the elements of the database. The comparison in this technique is done between original multimedia data and prototypical category such as clothes, vehicles, etc. [11]. This technique based on semantic equivalence instead of syntactic equivalence between different queries [12]. The retrieval process can be optimized by combining the query and data-centric method [7]. Using this technique in image retrieval, depend on extract the image metadata prior in the database, as well putting semantics of query and images in the same category, in order to facilitate defining similarity by the query processor [13]. The process of extracting semantic data can be divided into five steps that are shown in [13].

\subsubsection{Metadata}

Metadata is the semantic information used to classify the characteristics of the data such as content, condition, quality, etc. [14]. The search varies; it uses conceptual level rather than normal string because users search for semantic contents of the data [15]. Increasing the representation of metadata is useful for many functions like data searching, data summarizing to simpler formats, and connecting with other kinds of sources [15]. There are multiple schemes of metadata; the most popular schemes are Dublin Core, MPEG-7, and IEEE LOM. 


\section{Background}

The multimedia objects are stored as digitized representations in the content-based retrieval (CBR) applications. The queries in such system can be fuzzy and ambiguous. Consequently, there are several factors that influence the success measure of the system depends on the indexing scheme and the system itself.

In particular, the content-based image retrieval architecture is shown in Fig. 2. The system consists of two parts; each part is surrounded by a rounded rectangle. The right side represents the data insertion subsystem that is responsible for storing images in the image database as well as extracting features from these images and storing the extracted features in the feature database. Whereas the left side is responsible for query processing; first, a user specifies the query by an image. Then, the module extracts the feature vector from a query image. After that, the module evaluates the similarity between the query image and the database images by applying some metric such as Euclidean distance. Finally, the similar images are retrieved to the user. Indeed, to speed up the similarity computation and retrieval, the database images are indexed by using structures like M-tree or Slim-tree according to feature vectors of the images.

Relevance feedback is an interactive method used to enhance the effectiveness of retrieval systems. The method goes through a number of steps, which are: (1) the system searches and returns a small set of images similar to the user query pattern, (2) the user marked which images are relevant to the query image, (3) the system reformulates automatically the query depend on relevance judgments of the user. The process continues until the user is satisfied.

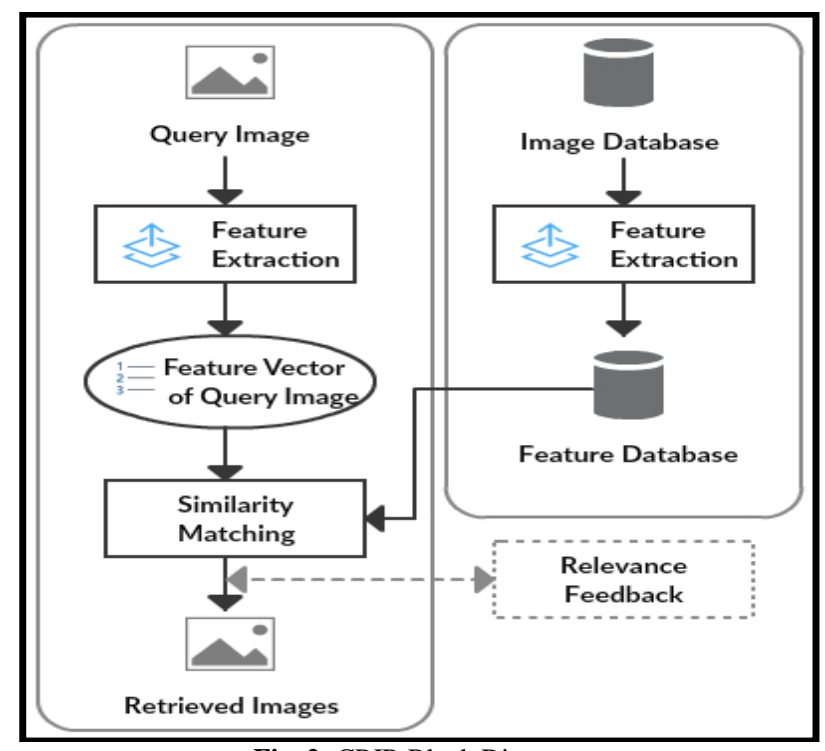

Fig. 2: CBIR Block Diagram.

\subsection{CBR for images}

The image content consists of several objects; some of these objects are substantially from the system perspective. They have different associated properties such as the descriptor of features that define the characteristics of a pixel or set of pixels, e.g. RGB values. And descriptor of form or location, that specifies the location of the object within the image [3].

The images are divided into a group of rectangular equal-sized cells that form the image grid, where the homogeneous regions are called segments. A region $\mathrm{R}$ is set of cells $\mathrm{C} 1, \mathrm{C} 2, \ldots, \mathrm{Cn}$ where the Euclidian distance between two consecutive cells is 1, if $\left(\mathrm{x}_{1}, \mathrm{y}_{1}\right) \in \mathrm{R}$, and $\left(\mathrm{x}_{2}, \mathrm{y}_{2}\right) \in \mathrm{R}$ exist, then $\mathrm{C}_{1}=\left(\mathrm{x}_{1}, \mathrm{y}_{1}\right)$, and $\mathrm{C}_{\mathrm{n}}=\left(\mathrm{x}_{2}, \mathrm{y}_{2}\right)$. Each image is associated with a homogeneity predicate, which is a function $\mathrm{H}$ that takes a region $\mathrm{R}$ as input and return true or false e.g. H returns true if having more than $100 \mathrm{x} \delta \%$ cells in the specified region have the same color, where $\delta \in[0,1][3]$.

In general, not all the pixels of an image must be considered. Therefore, the image matrix is transformed into a compressed representation, by using e.g., Discrete Fourier Transform (DFT), Wavelet, or Discrete Cosine Transform (DCT) technique [3].

In a case of two segments or images are similar, one of the similarity-based retrieval approaches is used. These approaches are: transformational (transformation cost based) and metrical (distance function based) [3].

\subsection{Applications}

The innovation of CBIR has been utilized in multiple applications, for example, fingerprint identification, telemedicine, digital libraries, crime prevention, biodiversity information systems and many other applications. In this section, some of these applications are presented.

\subsubsection{Digital libraries}

Many of digital libraries use content-based search services. One of these libraries is a digital museum of Taiwanese butterflies [16], this library contains a module that supports CBIR based on patters, texture, and color. Zhu et.al, [17], builds a library that aimed to retrieve geographical images. The system retrieves air photos based on texture descriptors. With each retrieved image, the place name is attached and can be presented by Geographical Name Information System (GNIS).

\subsubsection{Biodiversity information systems}

These systems help to develop and complete knowledge of the researchers, as well as comprehension of species and natural surroundings them through integrating textual and image content-based. Such of these systems is a library for biodiversity information systems [18] that showed the query specification and retrieving on some fish images. 


\subsubsection{Medical applications}

The CBIR technology can be utilized in multiple aspects such as diagnostic, research or teaching [19]. In some cases, clinicians make decisions by using similar cases for case-based reasoning. While textual data can be used to retrieve helpful information that may contain visual features or interesting images.

\section{Related work}

MPEG-7 [20] or Multimedia Content Description Interface describes the content of multimedia like image, speech, video, graphics and combinations of these components. One of the MPEG-7 components is an image descriptor, where a number of histogram descriptors, a color layout descriptor, and a dominant color descriptor are defined for the color property. For texture, there are several descriptors which are homogeneous texture descriptor, texture browsing descriptor, and local edge histogram descriptor. For shape, CSS descriptor is used. Tamura et.al, [21] proposed a texture signatures example.

\section{Research problem}

Due to the development of internet and computing technologies, multimedia structure greatly affects our life. That is the reason of finding another procedure to effortlessly retrieve tremendous multimedia information and files anytime is high demand. Some user queries in the multimedia database can return inaccurate answers or even not return any answers, because a response to a multimedia query is a complex process for the user to investigate. Thereby, there are multiple factors that affect the accuracy of these systems obviously. The impact of some of these factors on such systems will be addressed and analyzed to facilitate the selection of appropriate standards while building such applications.

\section{Analysis}

The image descriptor has two functions: the feature extraction and the distance function used in indexation of images by similarity. The function of feature extraction produces feature vector, which forms the image properties. The distance function calculates the dissimilarity between two images based on their properties. There are several features of images that can be used to identify the similar images in retrieving processes such as color, shape, texture and other related objects such as the use of object boundary and its features e.g. boundary length.

\subsection{Color descriptors}

The most common visual feature in CBIR systems, there are three areas in this field which are: (1) defining a sufficient color space for a specified target application, (2) choosing the proper extraction algorithm, (3) evaluating the similarity measures.

The information of color is represented as points in 3D color space such as RGB, YIQ, HSV, L*a*b*, L*a*v*. These spaces allow the similarity judgment and identification in addition to distinguishing between color stimuli. RGB and CMY color spaces are hardwareoriented because they consider the devices' properties used to reproduce colors. $\mathrm{L}^{*} \mathrm{a} \mathrm{b}^{*}$ and $\mathrm{L} * \mathrm{u} \mathrm{v}^{*}$ are user-inspired because they identify color differences as perceived by humans [22].

There are various techniques of color description; some of these techniques are encoding information of color spatial distribution. While the other techniques including Color Histogram and Color Moments. The extraction algorithm of Color Histogram consists of three steps, which are: (1) dividing the color space into cells, (2) connect each cell with a histogram bin, (3) for each cell, counting the number of image pixels and store this count in the histogram bin. Whereas, the feature vector in Color Moments is formed by the (1) mean, (2) variance, (3) skewness. On the contrary, the techniques that encoding the spatial distribution are included Color Coherence Vector (CCV), Color Correlogram and Border/Interior Pixel Classification (BIC). In CCVs, the coherent pixels $\left(\alpha_{\mathrm{i}}\right)$ and incoherent pixels $\left(\beta_{\mathrm{i}}\right)$ are calculated for each color, where the coherent pixel is a pixel that belongs to a largely uniformly-colored region. The Color Correlogram encodes the colors spatial correlation, it is represented as an ordered table by a color pair $\langle\mathrm{i}, \mathrm{j}\rangle$ and $\mathrm{k}$, where $\mathrm{k}$ indicates the probability of finding a pixel with color $\mathrm{j}$ at a distance $\mathrm{k}$ from a pixel with color $\mathrm{i}$. In BIC, the image pixels are partitioning into a border or interior pixels [22].

\subsection{Shape descriptors}

The shape property is important in pattern recognition; these descriptors are categorized into region-based and boundary-based or contourbased methods. Each of these methods is classified into global and structural or local descriptors. In global, the shape is represented as a whole, wherein local, the shape is represented as sections. Some of these shape descriptors are Moment Invariants, Beam Angle Statistics (BAS), Curvature Scale Space (CSS), Tensor Scale Descriptor (TSD), Segment Saliences (SS), and Contour Saliences (CS). More details about each of these shape descriptors can be found in [22].

\subsection{Texture descriptors}

This image property defines some visual patterns of an image such as granularity, repetitiveness, and directionality, which can be characterized by the presence of fundamental primitives whose spatial distribution makes these patterns. There are multiple approaches used to represent and extract textures, e.g. frequency-based, space-based and texture signatures. Frequency-based includes Gabor wavelet coefficients. In space-based model, Co-occurrence matrix is a technique used to encode texture information, by depicts the spatial relationships among grey-levels in an image, e.g. a cell at position ( $i, j)$ in a matrix stores the probability of having two pixels of gray levels $i$ and $j$ in two relative positions, then group of co-occurrence probabilities such as contrast, energy, and entropy is used to define textured regions. In texture signatures, the texture specified in terms of coarseness, contrast, and directionality [22]. 


\section{Experiment results}

An illustration of how different techniques, methods or descriptors can be instantiated, several experiments was conducted to evaluate the effectiveness achieved by different CBIR systems.

As mentioned before, in retrieval process the images are retrieved depend on the similarity measure. The similarity includes searching the database for similar coefficients that is done by using for example Euclidean and quadratic distance or any other method. The retrieval images are a list ranked by the similarities measure according to the query image. The images are ranked in ascending order.

In general, there are two criteria for measuring the success of the CBIR systems, which are: (a) precision P that measures the accuracy of the retrieval process, $\mathrm{P}$ is defined as in Eq. (1),

$$
\mathrm{P}=\frac{\mathrm{r}}{\mathrm{n}}
$$

Where $\mathrm{r}$ is a number of relevant retrieved images and $\mathrm{n}$ is the total number of retrieved images. (b) Recall $\mathrm{R}$ that measures the robustness of the retrieval process, $\mathrm{R}$ is defined as in Eq. (2),

$$
\mathrm{R}=\frac{\mathrm{r}}{\mathrm{m}}
$$

Where $\mathrm{r}$ is the number of relevant retrieved images as said before and $\mathrm{m}$ is the total number of relevant images in the whole database. In order to evaluate the performance of some CBIR systems that was programmed using Matlab, the Matlab version R2016b was used in experiments; multiple experiments were conducted on WANG database [23], [24]. The WANG database consists of 10 classes, in each class there are 100 images. The 10 classes are utilized for estimation of relevance: according to a query image, assuming the user searches for images from the same class, therefore the rest 99 images from a class of the query image are considered relevant while the remaining images in other classes are considered irrelevant.

The first experiment [25] aimed to compare between images indexing and retrieval when using clustering techniques in a discrete cosine transform (DCT) domain and moments. Fig. 3 shows the retrieval results when using moments, while Fig. 4 shows the retrieval results when using clustering. As a result, moments proved to be more successful than clustering in this software.

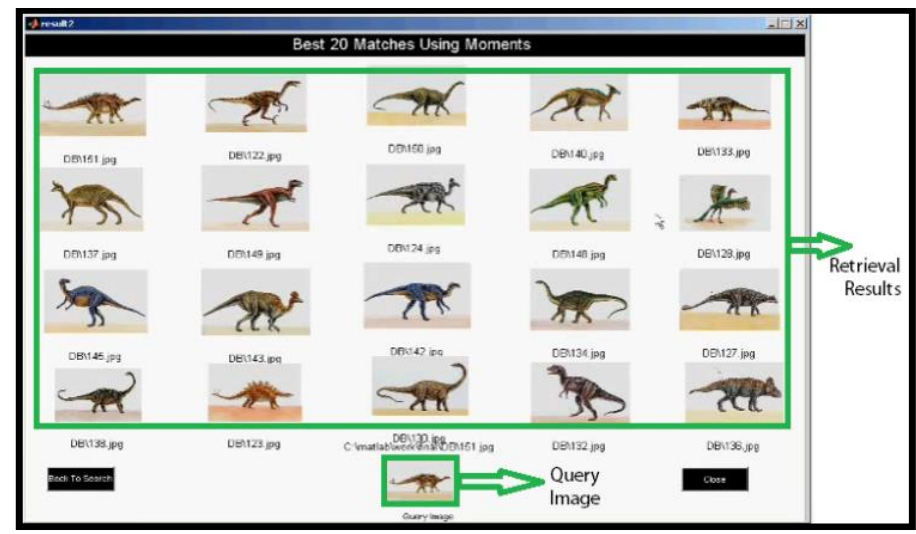

Fig. 3: Searching Using Moments.

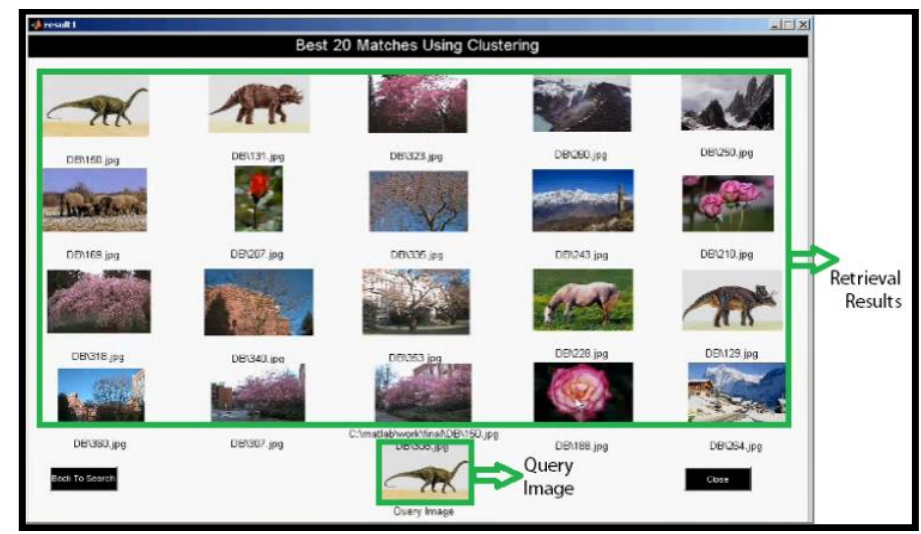

Fig. 4: Searching Using Clustering.

The second experiment [26] compares between global and local image descriptors but the goal is to find the combination of local features that produce the best results. Of course, global features better because the local method cannot capture all the keywords, especially when having a large dictionary, which gives a slower system and weakens the distance computation. From 64 local options, the generated local feature that gave the best results when concatenating Color Histogram with 32 bins and color space RGB with EHD. F-measure is one of the measures that combine precision and recall together, in this software, f-measure for 64 local options was presented in Fig. 5. 


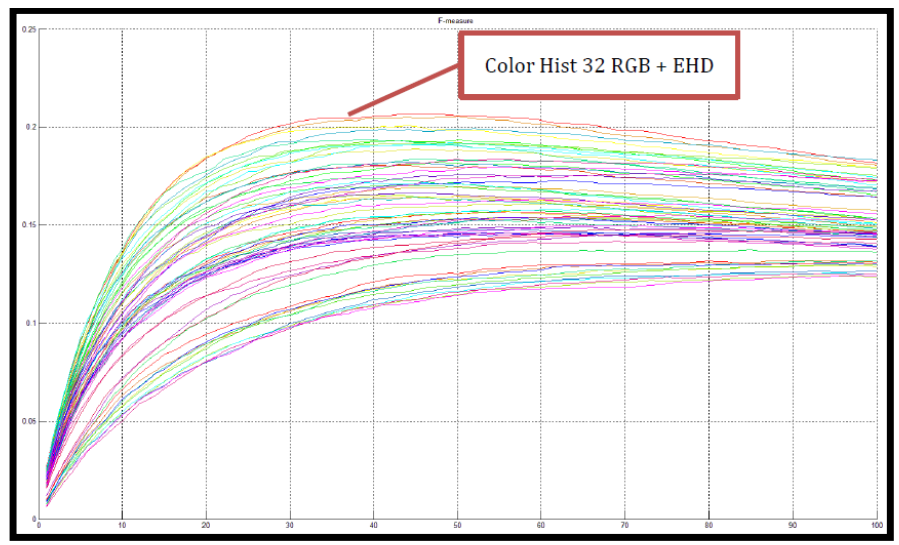

Fig. 5: F-Measure of Local Features [26].

Furthermore, the recognition engine or device is used to classify the patterns. It can be probabilistic, rule-based, or a combination of them. Some of the popular classifiers are K-Nearest Neighbor (KNN), Support Vector Machine (SVM), Artificial Neural Network (ANN), Hidden Markov Model (HMM), etc. The selection of the classifier can affect directly the recognition accuracy of the system. In the third experiment [27], the CBIR software uses two classifiers, which are KNN and SVM. The accuracy of the software when using each one of these classifiers is shown in Fig. 6. As you shown, SVM is better than KNN.

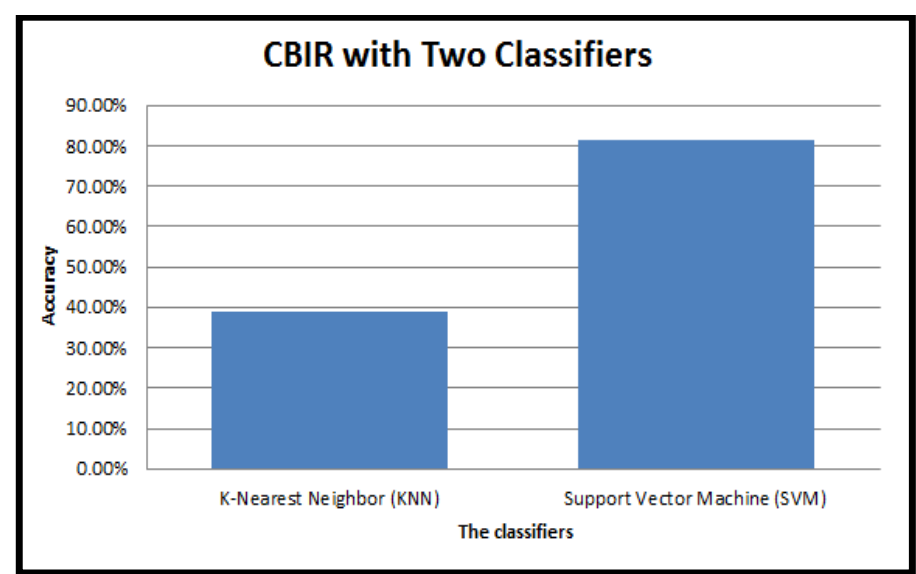

Fig. 6: The Accuracy of CBIR System Using KNN and SVM Classifiers.

\section{Conclusion}

This paper has showed a review in the field of content-based image retrieval. Firstly, a set of constructs identifying the primary related concepts precisely. Next, describing the essential problems needs to consider while designing these kinds of image retrieving systems by defining suitable image descriptors.

In CBIR systems, the images are stored in digitized representations. The users' queries are characterized by ambiguity and fuzziness. There are two types of possible errors, which are the false results that returned as query solution, and absent results that should be returned but do not exist in the query response. Metrics as precision and recall or any other type of measures that combine precision and recall such as f-measure and accuracy used to evaluate the query results. Hence, some existing software is evaluated by these metrics in order to see the effect of each method while retrieving the similar images that was used in the systems.

\section{Future work and recommendations}

A lot of methods and approaches can be used to enhance retrieval process in CBIR systems. In this paper, one of the software uses the KNN and SVM classifiers. With using another type of classifiers that have proven the effectiveness of several recognition systems such as Hidden Markov Model and Artificial Neural Network may increase the accuracy of these systems. Then the achieved retrieval accuracy from ANN and HMM will be compared with SVM.

\section{References}

[1] En.wikipedia.org. (2017). Multimedia database. [online] Available at: https://en.wikipedia.org/wiki/Multimedia_database [Accessed 14 Mar. 2017].

[2] Peterindia.net. (2017). Multimedia Database. [online] Available at: https://www.peterindia.net/MultimediaDatabase.html [Accessed 19 Mar. 2017].

[3] Negoiţ̧ă, C., \& Vlădoiu, M. Querying and Information Retrieval in Multimedia Databases. Universității Petrol-Gaze Din Ploieşti 2006th ser. VIII, 2 .

[4] Andrade, H., Kurc, T., Sussman, A. and Saltz, J. (2004). Optimizing the execution of multiple data analysis queries on parallel and distributed environments. IEEE Transactions on Parallel and Distributed Systems, 15(6), pp.520-532. https://doi.org/10.1109/TPDS.2004.11.

[5] Sadat, A. and Lecca, P. (2009). On the Performances in Simulation of Parallel Databases: An Overview on the Most Recent Techniques for Query Optimization. 2009 International Workshop on High Performance Computational Systems Biology. https://doi.org/10.1109/HiBi.2009.25. 
[6] Chan, S., Qing Li, Yi Wu, and Yueting Zhuang, (2002). Accommodating hybrid retrieval in a comprehensive video database management system. IEEE Transactions on Multimedia, 4(2), pp.146-159. https://doi.org/10.1109/TMM.2002.1017730.

[7] Ruziana Mohamad Rasli, Su-Cheng Haw, and Chee-Onn Wong, (2010). A survey on optimizing video and audio query retrieval in multimedia databases. $20103 \mathrm{rd}$ International Conference on Advanced Computer Theory and Engineering(ICACTE). https://doi.org/10.1109/ICACTE.2010.5579154.

[8] Zhou, W., Dao, S. and Jay Kuo, C. (2002). On-line knowledge- and rule-based video classification system for video indexing and dissemination. Information Systems, 27(8), pp.559-586. https://doi.org/10.1016/S0306-4379(02)00018-2.

[9] Agrawal, R., Faloutsos, C. and Swami, A. (1993). Efficient similarity search in sequence databases. Foundations of Data Organization and Algorithms, pp.69-84. https://doi.org/10.1007/3-540-57301-1 5.

[10] Shenoy, S. and Ozsoyoglu, Z. (1989). Design and implementation of a semantic query optimizer. IEEE Transactions on Knowledge and Data Engineering, 1(3), pp.344-361. https://doi.org/10.1109/69.87980.

[11] Janghyun Yoon, and Jayant, N. (n.d.). Relevance feedback for semantics based image retrieval. Proceedings 2001 International Conference on Image Processing (Cat. No.01CH37205)

[12] Shen, H., Ooi, B. and Zhou, X. (2005). Towards effective indexing for very large video sequence database. Proceedings of the 2005 ACM SIGMOD international conference on Management of data - SIGMOD '05. https://doi.org/10.1145/1066157.1066240.

[13] Li, W., Candan, K., Hirata, K. and Hara, Y. (1997). A hybrid approach to multimedia database systems through integration of semantics and media-based search. Lecture Notes in Computer Science, pp.182-197. https://doi.org/10.1007/3-540-63343-X_47.

[14] Wichterich, M., Assent, I., Kranen, P. and Seidl, T. (2008). Efficient EMD-based similarity search in multimedia databases via flexible dimensionality reduction. Proceedings of the 2008 ACM SIGMOD international conference on Management of data - SIGMOD '08. https://doi.org/10.1145/1376616.1376639.

[15] Steinacker, A., Ghavam, A. and Steinmetz, R. (2001). Metadata standards for Web-based resources. IEEE Multimedia, 8(1), pp.70-76. https://doi.org/10.1109/93.923956.

[16] J. S. Hong, H.Y. Chen, and J. Hsiang. A Digital Museum of Taiwanese Butterflies. In Proceedings of the Fifth ACM Conference on Digital Libraries, pages 260-261, San Antonio, Texas, United States, 2000. ACM Press. https://doi.org/10.1145/336597.336694.

[17] Bin Zhu, M. Ramsey and Hsinchun Chen, "Creating a large-scale content-based airphoto image digital library", IEEE Transactions on Image Processing, vol. 9, no. 1, pp. 163-167, 2000. https://doi.org/10.1109/83.817609.

[18] R. Torres, C. Medeiros, M. Gonçcalves and E. Fox, "A digital library framework for biodiversity information systems", International Journal on Digital Libraries, vol. 6, no. 1, pp. 3-17, 2006. https://doi.org/10.1007/s00799-005-0124-1.

[19] H. Müller, N. Michoux, D. Bandon and A. Geissbuhler, "A review of content-based image retrieval systems in medical applications-clinical benefits and future directions", International Journal of Medical Informatics, vol. 73, no. 1, pp. 1-23, 2004. https://doi.org/10.1016/j.ijmedinf.2003.11.024.

[20] Shih-Fu Chang, T. Sikora and A. Purl, "Overview of the MPEG-7 standard", IEEE Transactions on Circuits and Systems for Video Technology, vol. 11, no. 6, pp. 688-695, 2001. https://doi.org/10.1109/76.927421.

[21] H. Tamura, S. Mori and T. Yamawaki, "Textural Features Corresponding to Visual Perception", IEEE Transactions on Systems, Man, and Cybernetics, vol. 8, no. 6, pp. 460-473, 1978. https://doi.org/10.1109/TSMC.1978.4309999.

[22] da Silva Torres, R. \& Falcão, A. X. (2006). Content-Based Image Retrieval: Theory and Applications.. RITA, 13, 161-185.

[23] Jia Li, James Z. Wang, "Automatic linguistic indexing of pictures by a statistical modeling approach," IEEE Transactions on Pattern Analysis and Machine Intelligence, vol. 25, no. 9, pp. 1075-1088, 2003. https://doi.org/10.1109/TPAMI.2003.1227984.

[24] James Z. Wang, Jia Li, Gio Wiederhold, "SIMPLIcity: Semantics-sensitive Integrated Matching for Picture LIbraries," IEEE Trans. on Pattern Analysis and Machine Intelligence, vol 23, no.9, pp. 947-963, 2001. https://doi.org/10.1109/34.955109.

[25] "Project Definition - CBIR", Sites.google.com, 2017. [Online]. Available: https://sites.google.com/site/cbirpro/project-definition. [Accessed: 19Mar- 2017].

[26] "aminert/CBIR", GitHub, 2017. [Online]. Available: https://github.com/aminert/CBIR. [Accessed: 19- Mar- 2017].

[27] C. Retrieval, "Content Based Image Retrieval - File Exchange - MATLAB Central", Mathworks.com, 2017. [Online]. Available: https://www.mathworks.com/matlabcentral/fileexchange/42008-content-based-image-retrieval. [Accessed: 18- Mar- 2017]. 